\title{
Design and Investigation of Corporate Feed $4 \times 2$ Square Array Microstrip Antennas at S-Band
}

\author{
Ogherohwo E.P. and Adeniran A.O. \\ Department of P/Applied Physics, Department of P/Applied Physics \\ University of Jos, Jos, Ladoke Akintola Univ. of Tech., \\ Plateau State, Nigeria.P.M.B 4000, Ogbomoso, Oyo State, Nigeria
}

\begin{abstract}
Wireless local area network (WLAN) applications nowadays has become more popular especially those operating in the Sband (2.0-4.5Ghz).This paper present the design and enhancement of gain and bandwidth of microstrip antenna using the Agilent layout and probe feed. The antenna were designed to operate at S-band $(2.0-4.5 \mathrm{GHz})$.The dimensions of each single element of the microstrip antenna, at the operating frequency was calculated using transmission line model and the S-parameters data were obtained using Agilent simulator. The scaling factor of 1.10 was considered for the design on FR4 substrate with $\varepsilon_{r}=4.77$ with thickness of $1.62 \mathrm{~mm}$ and $\delta=0.019$ and proved to be operating with adequate bandwidth and radiation characteristics.
\end{abstract}

Keywords: Agilent(ADS), Probe feed, FR4and Microstrip.

\section{INTRODUCTION}

Microstrip patch antennas have attracted widespread interest due to their small size, lightweight, low profile, and low cost as well as to the fact that they are simple to manufacture,suited to planar and non-planar surfaces, mechanically robust, easily integrated with circuits, allow multifrequency operation to be achieved [1].

However, their further use in specific systems is limited because of their relatively narrowbandwidth. Inprincipal, wide bandwidth of microstrip patch antennas (MPAS) or bandwidth enhancement can be achieved by several efficient approaches [2], namely (i) increasing the substrate thickness (ii)optimizing impedance matching (iii)reducing the substrate effective permittivity or (iv)incorporating multiple resonance. Much effort has also been increasingly devoted to increasing the frequency agility of (MPAs)[2].

At the same time, MPAs need to be extremely small and compact to satisfy the severe size constraints of some critical applications such as mobile cellular handsets, card less phones and blue tooth devices. The miniaturization of normal MPA size (i)has typically been accomplished by loading ; which can take various forms, such as (i)using a high permittivity substrates ,(ii)Using shorting ports or shorting pins ,or (iii)modifying the basic patch shape[3].

In this paper, probe line feed $[4,5]$ techniques are applied to the square microstrip patch antenna this is because it is easy and can easily be placed at any point on the patch, it has low spurious radiation.

\subsection{Methods of Analysis}

There are three popular models for the analysis of microstrip antennas - viz: transmission line model, cavity model and full wave model. The transmission line model is the simplest. It gives a good physical insight but is less accurate. The cavity model, which is used in this work, is quite complex but gives good physical insight and is more accurate. The full wave model is the most complex. It is very accurate in the design of finite and infinite arrays or stacked structures.

The quantity associated with radiated EM wave is the Poynting vector given as: [18]

$S=E \times H(1)$

Where $\mathrm{S}$ is instantaneous Poynting vector, $\mathrm{E}$ is instantaneous electric field intensity and $\mathrm{H}$ is instantaneous magnetic field intensity. The complex fields $\mathrm{E}$ and $\mathrm{H}$ are related to their instantaneous counterparts by [17]:

$$
\begin{aligned}
& \left.\begin{array}{l}
E(x, y, z, t)=\operatorname{Re}\left[E(x, y, z) e^{i w t}\right] \\
H(x, y, z, t)=\operatorname{Re}\left[H(x, y, z) e^{i w t}\right]^{(2)}
\end{array}\right\} \\
& \text { Using and identity } \\
& \operatorname{Re}\left(X c^{i w t}\right)=\frac{1}{2}\left(X e^{i w t}+X^{*} e^{i w t}\right) \text { equation (1) can be }
\end{aligned}
$$
rewritten as;

$$
S=\frac{1}{2} \operatorname{Re}\left[E \times H^{*}\right]+\frac{1}{2} \operatorname{Re}\left[E \times H e^{i w t}\right]
$$

Hence, the time average Poynting vector can be written as [18].

$$
S_{a v}=\frac{1}{2} \operatorname{Re}[E \times H] W m^{-2}
$$

The factor $1 / 2$ appears because the $\mathrm{E}$ and $\mathrm{H}$ fields are peak values and not rms.This research aims to design and implement a circular microstrip patch antenna suitable for use at microwave frequencies.

\subsection{Radiation Mechanism}

Microstrip antennas are essentially suitably shaped discontinuities that are designed to radiate. The discontinuities represent abrupt changes in the microstrip line geometry. Discontinuities alter the electric and magnetic field 
distributions. These results in energy storage and sometimes radiation at the discontinuity.

As long as the physical dimensions and relative dielectric constant of the line remains constant, virtually no radiation occurs. However the discontinuity introduced by the rapid change in line width at the junction between the feed line and patch radiates. The other end of the patch where the metallization abruptly ends also radiates. When the field on a microstrip line encounters an abrupt change in width at the input to the patch electric fields spread out [6].

\subsection{Microstrip Lines}

A microstrip line consists of a single ground plane and a thin strip conductor on a low loss dielectric substrate [11] above the ground plate. Due to the absence of the top ground plate and the dielectric substrate above the strip, the electric field lines remain partially in the air and partially in the lower dielectric substrate.

This makes the mode of propagation not pure TEM but what is called quasi-TEM. Due to the open structure and any presence in discontinuity, the microstrip line radiates electromagnetic energy. The use of thin and high dielectric materials reduces the radiation loss of the open structure where the fields are mostly confined inside the dielectric.

\subsection{Quasi TEM Mode of Propagation}

The electromagnetic waves in free space propagate in the transverse electromagnetic mode (TEM). The electric and magnetic fields are mutually perpendicular and further in quadrature with the direction of i.e. along the transmission line Coaxial and parallel wire transmission line employ TEM mode. In this mode the electromagnetic field lines are contained entirely within the dielectric between the lines.But the microstrip structure involves an abrupt dielectric interface between the substrate and the air above it. Any transmission line system which is filled with a uniform dielectric can support a single well defined mode of propagation at least over a specific range of frequencies (TEM for coaxial lines TE or TM for wave guides).

Transmission lines which do not have such a uniform dielectric filling cannot support a single mode of propagation. Microstrip falls $[7,8]$ in this category. Here the bulk of energy is transmitted along the microstrip with a field distribution which quite closely resembles TEM and is usually referred to as Quasi - TEM. The microstrip design consists of finding the values of width (w) and length (l) corresponding to the characteristic impendence $\left(Z_{o}\right)$ defined at the design stage of the network. A substrate of permittivity $\left(\epsilon_{\mathrm{r}}\right)$ and thickness $(\mathrm{h})$ is chosen.

The effective microstrip permittivity $\left(\epsilon_{\text {eff }}\right)$ is unique to a fixed dielectric transmission line systemand provides a useful link between various wave lengths impedances and velocities. The microstrip in general, will have a finite strip thickness" which influences the field distribution for moderate power applications. The thickness of the conducting strip is quite significant when considering conductor losses [8]. For microstrip with $\mathrm{t} / \mathrm{h} \leq 0.005,2 \leq \mathrm{er} \leq 10$ and $\mathrm{w} / \mathrm{h} \geq 0.1$, the effects of the thickness are negligible. But at smaller values of $\mathrm{w} / \mathrm{h}$ or greater values of $\mathrm{t} / \mathrm{h}$ the significance increases.

\section{ANTENNA DESIGN PRINCIPLES}

The designed antenna is $4 \times 2$ elements a linear array.The first step of the design was the specification of a single microstrip patchantenna .The patch conductor can be assumed at any shape, but generally simple geometries are used, and this simplifies the analysis and performance prediction. Here the half wavelength rectangular patch element is chosen as the array element (as commonly used in microstrip antennas) [9].Its characteristics parameters are the length L,the width w, and the thickness h.To meet the initial design requirements (operating frequency $=$ between $2-4 \mathrm{GHz}$, andbeam with 9 degree) various analytical approximate approaches may be used. Here, thecalculations are based on the transmission line model[12].

Although, not critical, the width $\mathrm{w}$ of the radiating edge is specified first. The square patch geometry is chosen since it can be arranged to produce circularly polarized waves. Inpractice, the length $\mathrm{L}$ is slightly less than a half wavelength (in the dielectric).the length may also be specified by calculating the half wavelength value and then subtracting a small length to take into account the fringing fields [15-17].

\subsection{Square Patch Design Calculations}

The design of the square shape patch follows the equation for designing the rectangular shape patch. The same length and width of the patch of the antenna was made to ease the design steps, probe feed is being introduced into the design to offset the feeding location to the point where matched impedance can be achieved.The basic parameters of the microstrip has to be determined such the width and length and the feeding techniques that is beingused. Thewidth of the patch can be determined using equation 1 .

$$
W=\frac{1}{2 f\left(\sqrt{\varepsilon_{o} \mu_{o}}\right)} \sqrt{\frac{2}{\varepsilon_{r}+1}}(2)
$$

The $\varepsilon_{o}$ and $\mu_{o}$ are the permittivity and the permeability in free space respectively. The equation can be interpreted as the speed of light, $\mathrm{c}$ which is $3 \times 10^{8} \mathrm{~m} / \mathrm{s}$.

$$
\begin{aligned}
& f=\text { Resonant frequency } \\
& \varepsilon_{r}=\text { Relative permittivity of the dielectric material }
\end{aligned}
$$

The width of the antenna is used for the calculation of the antennas length. The patch length can be calculated using the equations [2].the lengths extension $\Delta L$ and the effective permittivity have to be calculated before calculation the length of the microstrip patch as shown in the figure below the $h$ is the height of the substrate while the $\mathrm{W}$ is the width of the patch as calculated before.

$$
L=\left(\frac{1}{2 f \sqrt{\varepsilon_{\text {reff }} \sqrt{\varepsilon_{o} \mu_{o}}}}\right)-2 \Delta L
$$




$$
\begin{gathered}
\Delta L=0.4212 h \frac{\left(\varepsilon_{\text {reff }}+0.3\right)\left(\frac{w}{h}+0.264\right)}{\left(\varepsilon_{\text {reff }}-0.258\right)\left(\frac{w}{h}+0.8\right)} \\
\varepsilon_{\text {reff }}=\left(\frac{\varepsilon_{r}+1}{2}\right)+\left[\left(\frac{\varepsilon_{\text {reff }}-1}{2}\right)\left[1+12 \frac{h}{w}\right]\right]^{-0.5}
\end{gathered}
$$

Where

$f=$ operating frequency

$$
\varepsilon_{e}=\text { permittivity of the dielectric }
$$

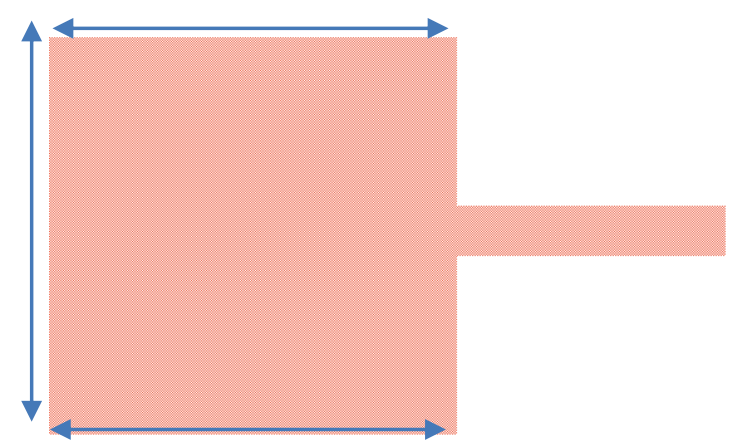

Figure 1: The simulated patch layouts

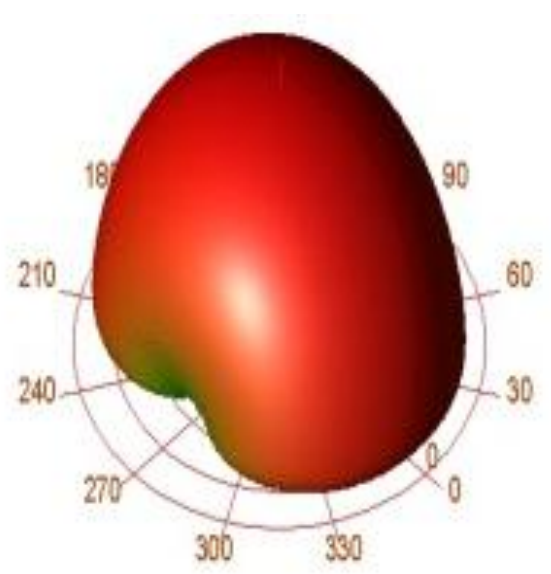

$\varepsilon_{\text {reff }}=$ effective permittivity of the dielectric

$\mu_{o}=$ permeability in free space

W=patch's width

$h=$ Thickness of the dielectric

Thewidth of the patch is made to be in the same value with the length to ease the design process, so the patch will be a square patch.Actually, the determination of the patch width is important because it affects the efficiency of the antenna.A small value of the width will leads to low antenna efficiency while high value of width will leads to higher order modes.(4).The type of feed employed in this research is probe fed which is easiest fed techniques .

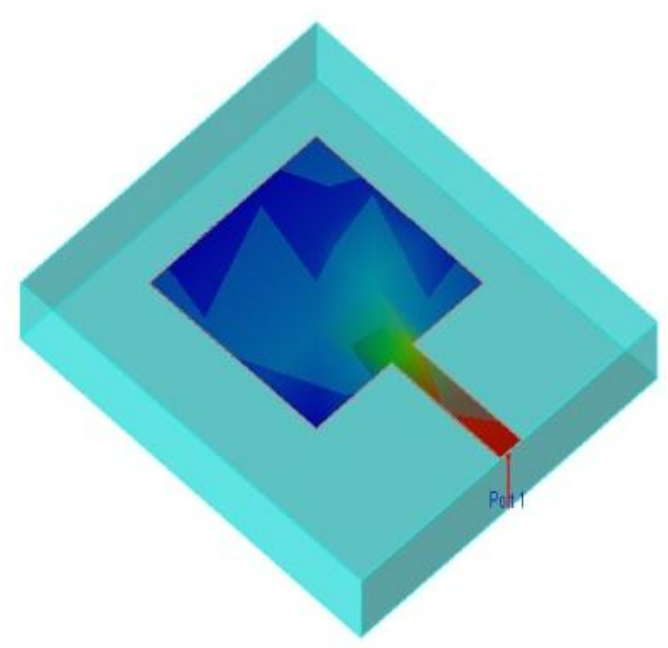

Figure 2: Single element onFR4 substrate

S11

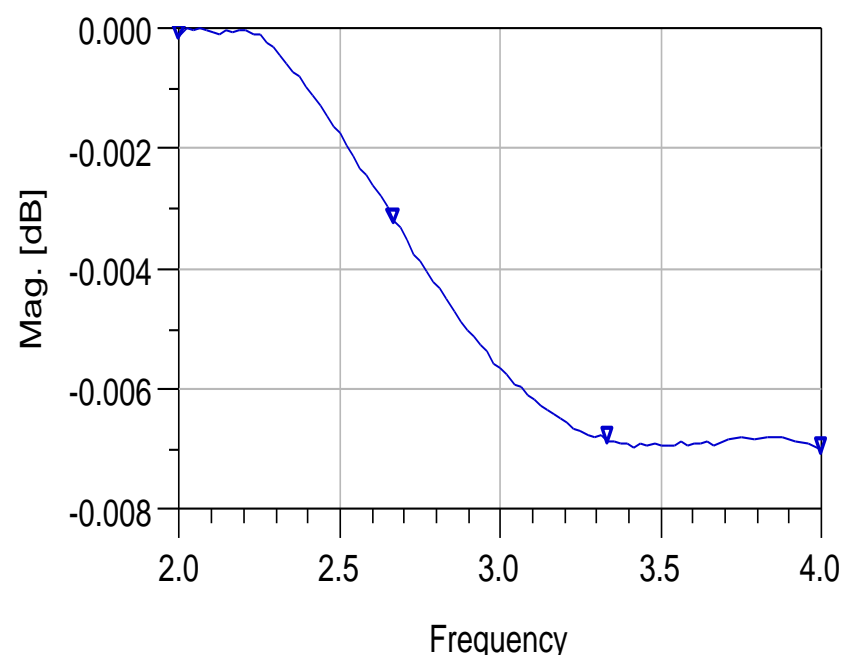

Figure 4: Return loss of Single element

Figure 3: 3D Radiation pattern for single element 


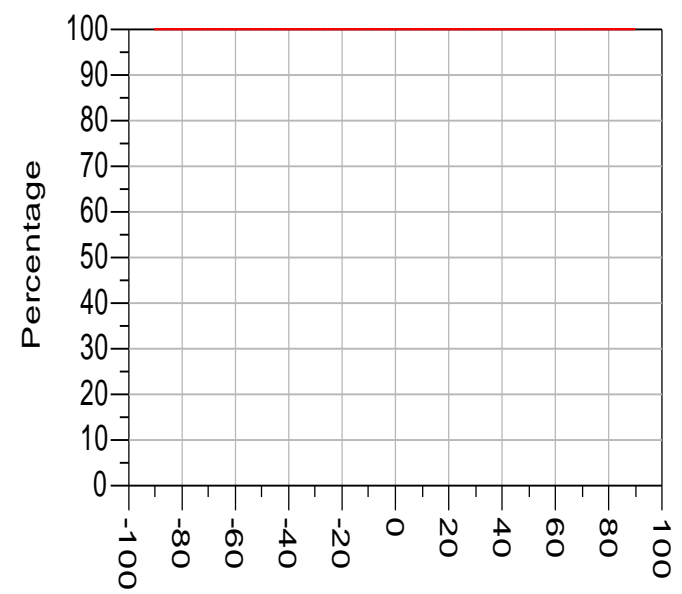

THETA

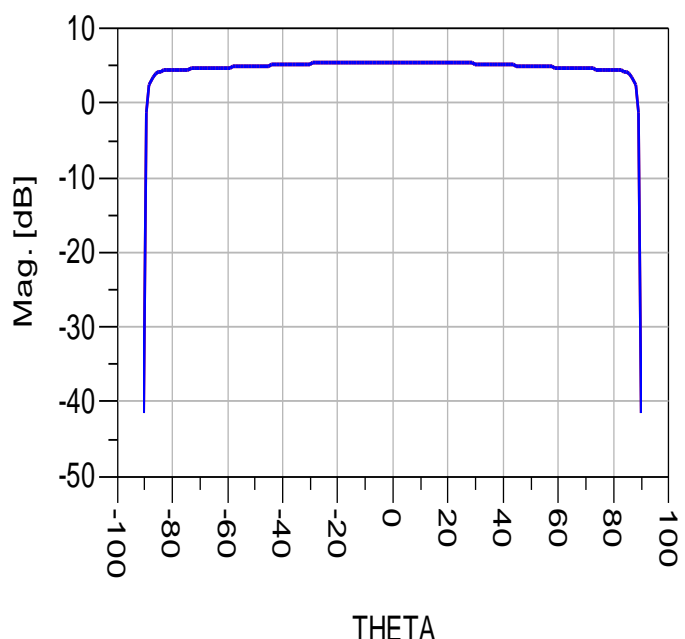

Figure 6:Gain of single element

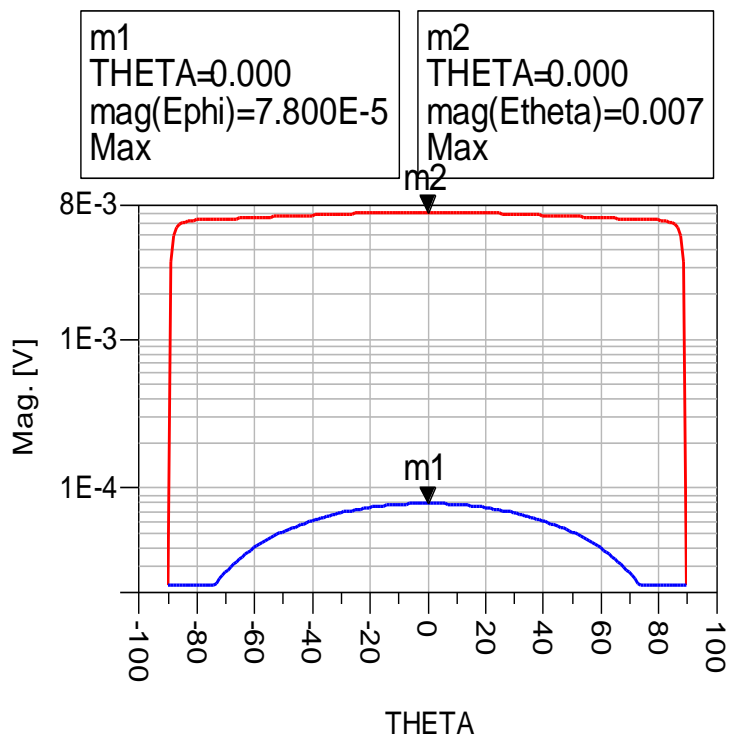

Figure7: Single Patch Antenna Radiation Pattern(2D)

\subsection{CorporateFeed Network}

Series feed microstrip array is formed by interconnecting all the element with high impedance transmission line and feeding the power at the first element .because the feed arrangement is compact the line losses associated with this type. The main limitation in series feed arrays is the large variation of the impedance and beam pointing direction over a band of frequencies[5].
The corporate feed network is used to provide power splits of 2 n(i.e. $n=2,4,8,16$,etc).This is accomplished by using either tapered lines or using quarter wavelength impedance transformer [5,6].In this paper the patch elements are connected by using the quarter wavelength impedance transformer method.

Corporate feed arrays are general and versatile. This method has more control of the feed of each element and is ideal for scanning phased arrays. Multiband arrays. Thus it provides better directivity as well as radiation efficiency and reduce the beam fluctuations over a band of frequencies compared to 
the series feed array [5,9].the phase of each element can be controlled by using phase shifters while amplitude can be adjusted using either amplifiers or attenuators $[2,8]$.

In this paper we have investigated the performance of corporate feed array in case of square patch elements.

\subsection{Square Patch Microstrip Array}

In this paper we have designed and simulated the $4 \times 2$ elements square patch microstrip array antenna using ADS2009 as shown in Figure 4, to cover S band frequency $(2 \mathrm{GHz}-4 \mathrm{GHz})$ operating frequencies .Here the power is fed to the antenna by using the Microstrip transmission line method[2,3] and the patch elements are matched together as well as with the transmission line.

$$
\theta=\text { Azimuth angle measured in degree }
$$

$k_{0}=$ wavelength of free space $\left(2.99 \times 10^{10}\right)$

$\mathrm{w}=$ Width of the patch $0.001 \mathrm{~m}$

The array factor is given in $[4,8]$ as

$$
F A=\frac{\sin ^{2}\left(N \pi\left(\frac{d_{x}}{\lambda}\right) \sin \theta\right)}{N^{2} \sin ^{2}\left(\pi\left(\frac{d_{x}}{\lambda}\right) \sin \theta\right)}
$$

The radiated field of the E-plane for a single element square patch can be expressed by adopting rectangular equation formula givenas:

$$
E(\theta)=\frac{\sin \left(\frac{k_{\mathrm{o}} h}{2} \cos \theta\right)}{\frac{k_{\mathrm{o}} h}{2} \cos \theta} \cos \left(\frac{k_{\mathrm{o}} L}{2} \cos \theta\right)
$$

(6)

Where -:

$\mathrm{L}$ = length of the patch

$\mathrm{h}=$ substrate height of the patch

$d_{x}=$ Elements spacing

$\mathrm{N}=$ number of elements.

Usually the length of the $\boldsymbol{d}_{x}$ is made to be half wave length of the operating frequency of the element as shown in equation (6).But to be more precise, in this project the length $\boldsymbol{d}_{x}$ is determined to make sure that the input impedance from the subsequent element $(m+1)$ will be open circuit (high impedance).[6].

$$
d_{x}=\frac{\lambda_{o}}{2}
$$

Here,
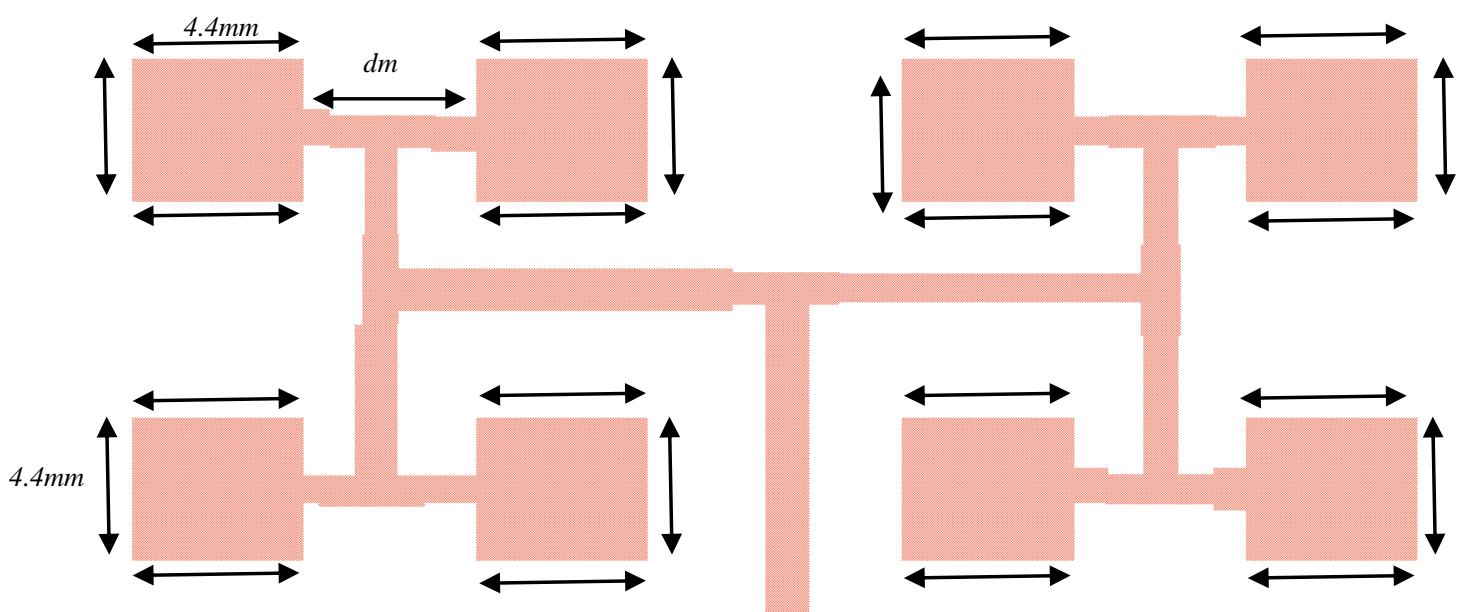


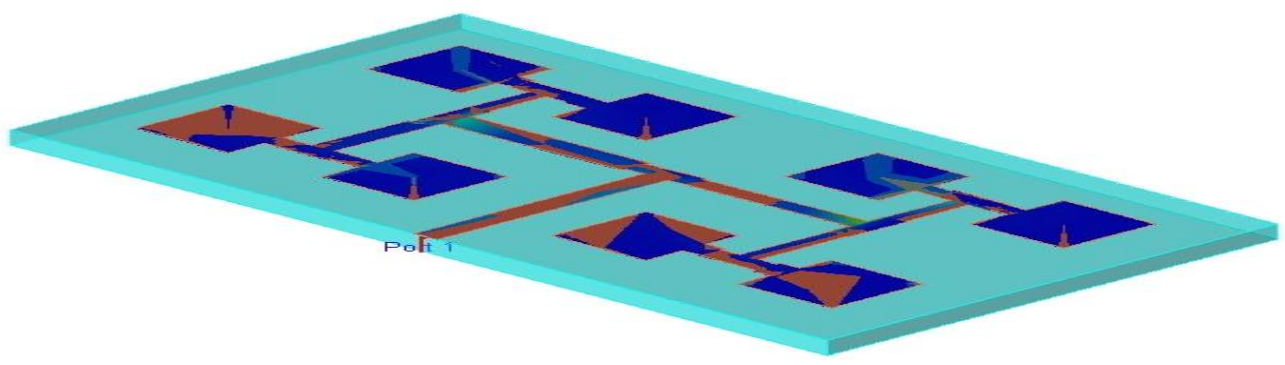

Figure 7:The simulated diagram of the Square Array Patch on ADS2009

\section{RESULTS AND DISCUSSION}

The designed and simulated square array patch antenna as shown in figure shows a narrow radiation at the frequency $2.99 \mathrm{GHz}$, after the simulation results the array antenna exhibit

\section{S11}

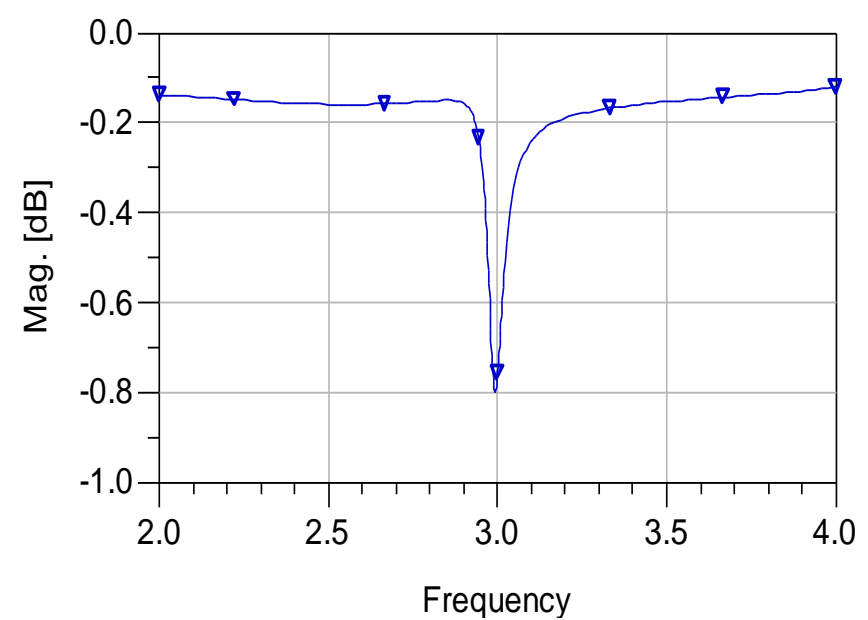

Figure 8:Return Loss in dB for array square

S11

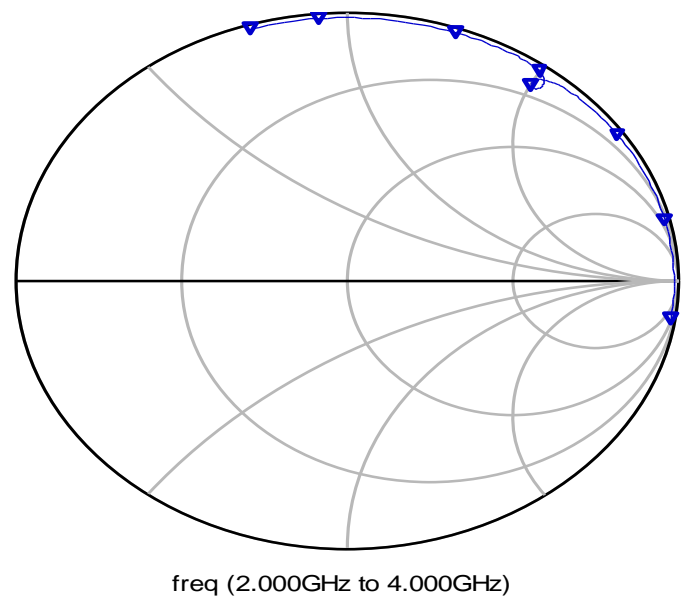

Figure 10: Showing the smith chart for the return loss for the array antenna at $2.9 \mathrm{GHz}$ a return loss of $-0.8 \mathrm{~dB}$ and gain of single was low and there is need for increase in the gain which lead to the arraying of square patch to increase the gain and enhance the bandwidth for the patch antenna. The value obtained as shown in the table 1 below shows the comparison from the single and array patch

antenna.

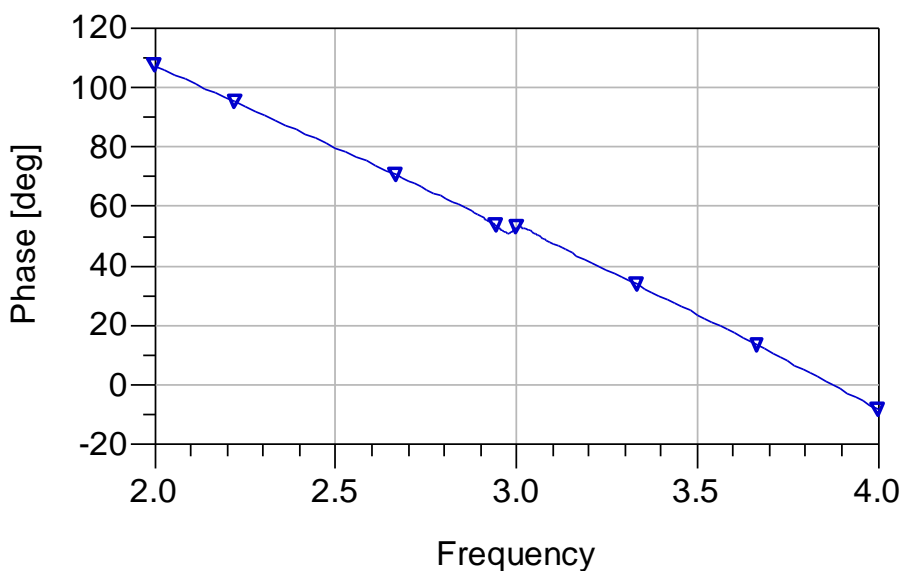

Figure 9: Showing the Phase angle in degree against frequency in $\mathrm{GHz}$

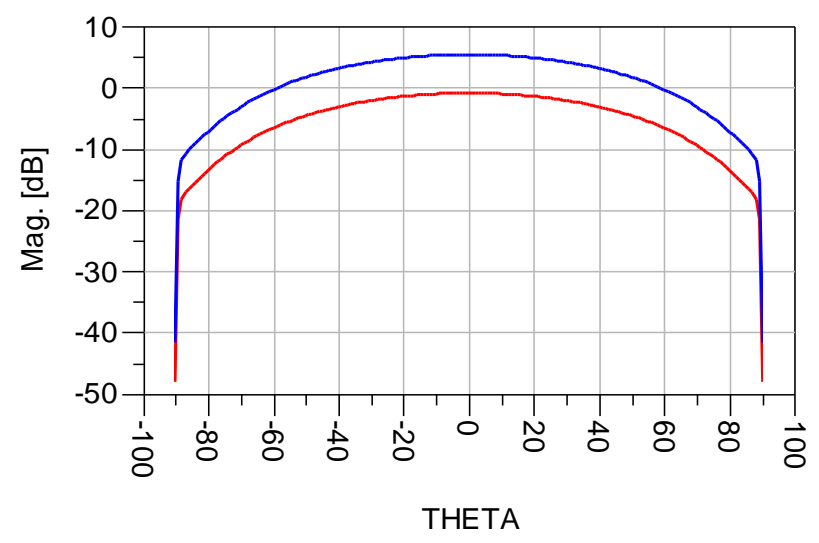

Figure 11: The Directivity and Gain in $d B$ 


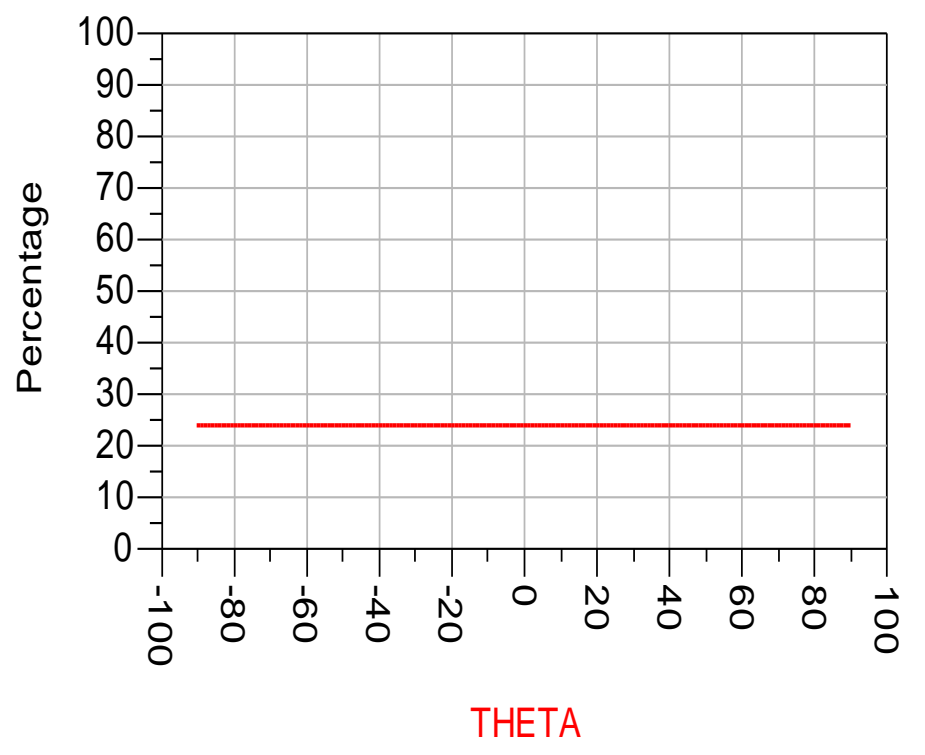

Figure 12: The percentage Efficiency of array

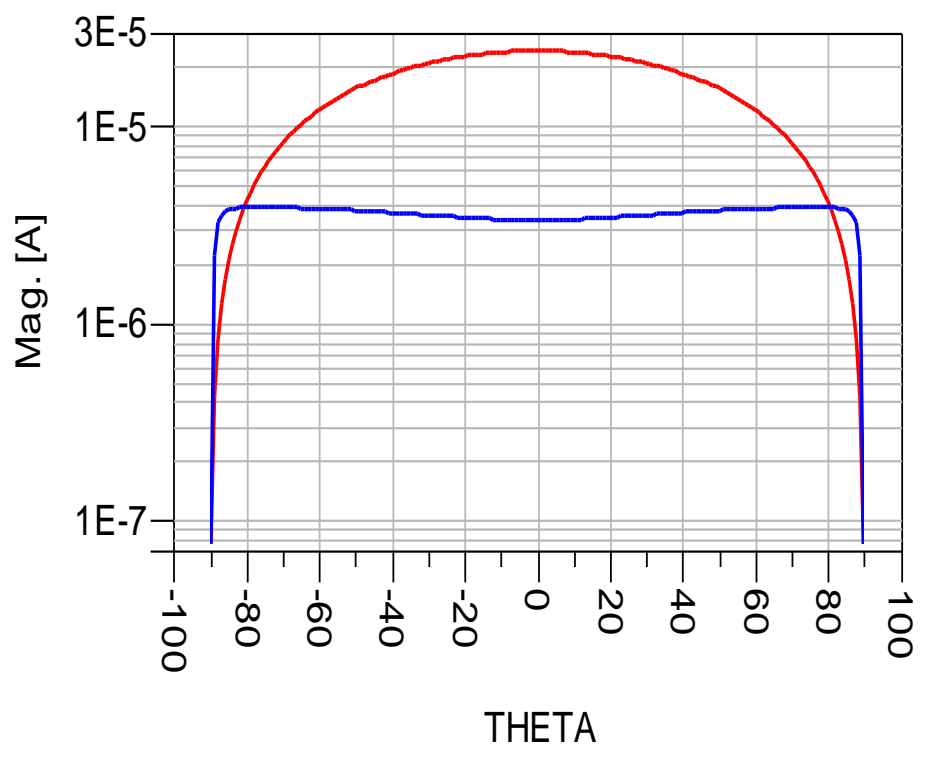

Figure 14: Showing $\mathrm{H}(\mathrm{phi})$ and H(theta) against the magnitude (Magnetic field)

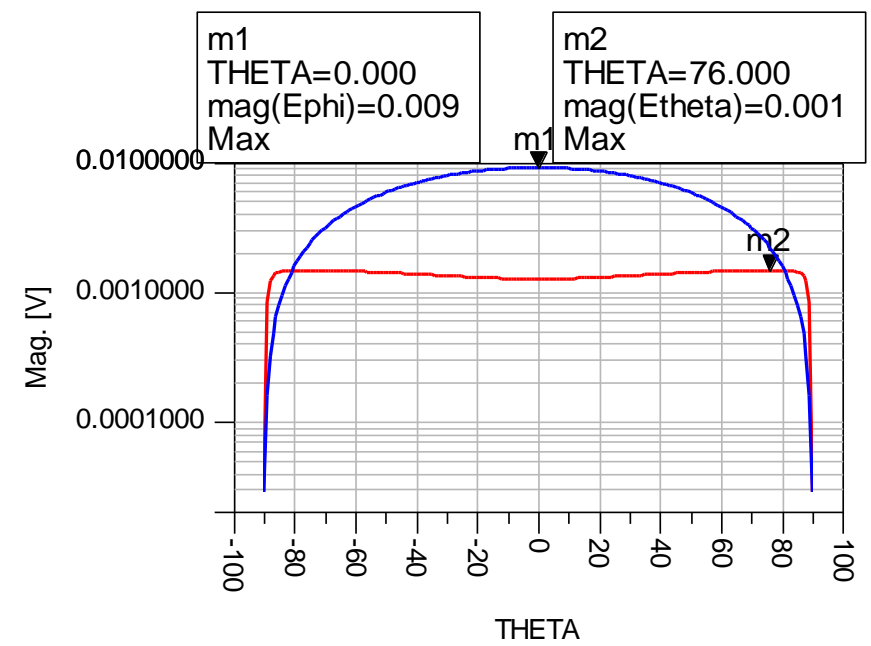

Figure 13: Showing E(Phi) and E(theta) against the magnitude (Electric Field)

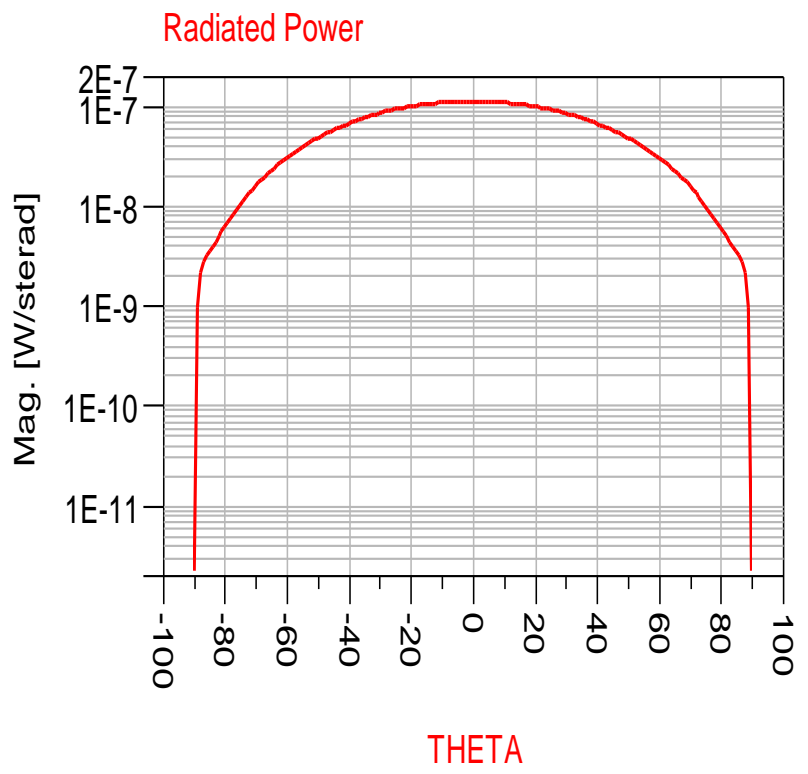

Figure 15: Showing the power radiated by Square array patch antenna 
Table 1: Antenna Parameters for the Single and Array Square Patch

\begin{tabular}{|c|c|c|}
\hline Parameters & $\begin{array}{c}\text { Single Element } \\
\text { (Simulated) }\end{array}$ & $\begin{array}{c}\text { Array Element } \\
\text { (Simulated) }\end{array}$ \\
\hline Gain(dB) & $2.12 \mathrm{~dB}$ & $9.5 \mathrm{~dB}$ \\
\hline Directivity & $0.245 \mathrm{dBi}$ & $15 \mathrm{dBi}$ \\
\hline Return loss & $-0.005 \mathrm{~dB}$ & $-0.8 \mathrm{~dB}$ at $2.99 \mathrm{GHz}$ \\
\hline Bandwidth & 1.86 & 34.32 \\
\hline Beam width & 50 degree & 10 degree \\
\hline Efficiency & $95 \%$ & $23 \%$ \\
\hline Radiated power & - & $1 \mathrm{E}-7 \mathrm{~dB}$ \\
\hline E-Phi & $7.89 \mathrm{E} 08$ & $0.009 \mathrm{~V}$ \\
\hline E-theta & 0.007 & $0.001 \mathrm{~V}$ \\
\hline H-Phi & - & $3.5 \mathrm{E}-5 \mathrm{~dB}$ \\
\hline H-theta & - & \\
\hline
\end{tabular}

\section{CONCLUSION}

A single and $4 \times 2$ square element array has been realized for wireless network at S-Band in this study.It consists of a single layer narrow band antenna element based on $4 \times 2$ element array was designed. Gain, Bandwidth, Effective Area,Directivity,Beamwidth,Radiation Pattern and the Return Loss for single and array patch are obtained and compared with each other over a frequency of $2.9 \mathrm{GHz}$.

From the data obtained,it has been pointed out that the side lobe level is the most critical factor, and thus determines the operating bandwidth.However,considering the impedance, gain and the lobe at $2.99 \mathrm{GHz}$ frequency the highest bandwidth was obtained.

\section{REFERENCES}

[1] James J.R., and Hall P.S., "Handbook of Microstrip Antennas" Peter Peregrinus Ltd., London, UK, 1989.

[2] Wong K.L, Compact and Broadband Microstrip Antennas, Wiley, New

[3] Row G.S,Yeh, and K. L. Wong, “Compact Dual Polarized Microstrip

[4] Advance Design System (ADS2009).

[5] Stutzman W.L, Thiele G.A, Antenna Theory and design, John Wiley \& Sons,

[6] Derneryd A, "Linearly Polarized Microstrip Antennas", IEEE Trans.Antennas and Propagation, AP-24, pp. 846-851, 1976.

[7] Rahim, M.K.A Wideband active antenna. University of Birmingham: Ph.D:Thesis.2003.
[8] Asrkinm.M.K.A, Rahim, M.H 'Square Shaped Dual Band Active Integrated Antenna and Passive Antenna 'International symposium on Antennas and Propagation ISAP 2006.

[9] Anitha M.K and Narayana 'Design of an 8x1 Square Microstrip Antenna Array 'International Journal of Electronic Engineering Research, Vol.1 Number 1(2009).pp71-77.

[10] Pozar D.M., and Schaubert D.H., "Microstrip Antennas, the Analysis and Design of Microstrip Antennas and Arrays", IEEE Press, New York, USA, 1995.

[11] CarverK.R and J.W.Mink, "Microstrip Antenna Technology", IEEE Trans. Antenna Propag., Vol.AP-29, pp.2-24, Jan 1981

[12] Bahl I.J. and P.Bhartia, "Microstrip Antennas", Dedham, MA: Artech House, 1980.

[13] James, J.R. P.S.Hall and Wood, "Microstrip Antenna Theory and Design", London, UK,: Peter Peregrinus, 1981.

[14] Amman M, Design of Microstrip Patch Antenna for the 2.4 Ghz Band, Applied Microwave and Wireless, pp. 24-34, November /December 1997. 2nd Ed., New York, 1998.

[15] Wong K. L., Design of No planar Microstrip Antennas and Transmission Lines, John Wiley \& Sons, New York, 1999.

[16] Stutzman W. L., G. A. Thiele, Antenna Theory and Design, John Wiley \& Sons,2nd Edition ,New York, 1998.

[17] Okwara, B. J. Kwaha and P. Amalu (2012) "Design and construction of array dipole antenna adaptable to VHF and UHF bands". Indian J. Sci. Technol. Vol. 4, Issue 7, pp. 736739.

[18] Balanis C.A. (1997). Antenna Theory Analysis and Design Handbook.Second Edition, United States of America, John Willey \& Sons Inc. New York. p. 734. 